\title{
Urgences
}

\section{L'image de soi}

\section{Jacques Daignault}

Numéro 27, mars 1990

Images imaginaires

URI : https://id.erudit.org/iderudit/025571ar

DOI : https://doi.org/10.7202/025571ar

Aller au sommaire du numéro

Éditeur(s)

Urgences

ISSN

0226-9554 (imprimé)

1927-3924 (numérique)

Découvrir la revue

Citer ce document

Daignault, J. (1990). L'image de soi. Urgences, (27), 35-38.

https://doi.org/10.7202/025571ar d'utilisation que vous pouvez consulter en ligne.

https://apropos.erudit.org/fr/usagers/politique-dutilisation/ 


\section{L'image de soi Jacques Daignault}

II n'est pas facile, sachez-le, pour un individu qui a passé sa vie à se distinguer, de finir chez Provigo.

Yves Boisvert ${ }^{1}$

\section{L'écriture sans traces}

Lire ce qu'on a déjà dit des mois ou même des années auparavant, en particulier ce que la parole avait échappé au profit de l'écriture - orale, cette fois -, c'est comme lire quelqu'un d'autre. Et la mémoire n'est pas en faute, ici; car il n'a jamais été dit qu'elle puisse enregistrer l'impossible. Or qu'est-ce que l'impossible pour elle? Penfield soutenait pourtant que le cerveau enregistre tout. Mais la mémoire estelle neuronale? Celle dont il est ici question serait plutôt à définir comme un approvisionnement de la conscience géré par le seul effort de se souvenir. Or de quoi est-il impossible de se souvenir, qui aurait été prononcé de parole vive? Confronté à la transcription d'un enregistrement, comme s'il s'agissait des mots, des images et des idées d'un autre, de quoi s'étonner alors? De deux choses, peut-être: de cela même qui est dit et du fait que ce soit bien de son cru.

Et s'il n'y avait rien là d'étonnant? Si au contraire il s'agissait bel et bien du texte d'un autre et qu'on en partageait à ce point le sens qu'il ne soit plus source aucune d'étonnement pour soi? La question ne serait plus la même. Comment quelqu'un d'autre a-t-il pu écrire exactement ce que je pense, et dans les termes mêmes où je l'aurais moi-même écrit? Voilà ce qu'il y aurait d'étonnant. Et pourtant.

Ce qu'il est impossible à la mémoire d'enregistrer, c'est précisément l'origine d'une pensée; autant dire la frontière même de soi. Impossible, en somme, de se souvenir de qui on est. Et de cela découle qu'il est également impossible de se

1 Chose Aucoin [Yves Boisvert], "La vie formidable d'un pseudocrate ", in Urgences, "Appellation contrólee ", numéro 20, Rimouski, 1988, p. 10. 
36

souvenir de ce que le soi, lui-même oublié, avait pu penser. En effet, de quoi aurait l'air un souvenir qui ne serait pas le sien? On ne se souvient jamais de ce qui est intimement lié à soi.

L'oubli de soi est le meilleur antidote contre la mort, son instinct dirait Freud: la répétition du même, le retour au repos originaire. La vie serait impossible, et dans tous les sens du mot, s'il n'y avait pas cette source même de l'étonnement qu'est l'oubli de soi. Le génie des différences croît bien dans l'impossibilité qu'a la mémoire d'enregistrer l'écriture.

Peu importe alors que l'écriture soit orale ou textuelle, le signe écrit - le signifiant du signifiant, comme on dit aujourd'hui - n'est pas une mémoire, ou même un aide-mémoire, mais sa limite. Phonétique ou grammatologique, l'écriture ne laisse aucune trace pour soi

L'écriture est le code même de l'oubli.

Tout cela n'est-il pas contraire à l'analyse ou à la généalogie : retrouver ce qui était devenu inconscient et rencontrer ses origines? Mais ce que restitue la mémoire est bien moins que ce qu'on en fait. L'analyse n'est pas réductible aux expériences, en laboratoire, sur la stimulation des neurones de la mémoire; non plus que l'histoire, à la série des faits. Le sens, toujours pour soi, est tout le contraire d'une trace. L'histoire aussi est vivante; et dans la mesure même où elle s'écrit: division cellulaire dans les faits d'armes ou les malheurs de soi. Le sens vaut mieux que l'origine; et l'oubli, que la trace. Tout meurt de mémoire.

L'écriture est biologique.

\section{Le travail ou l'écriture de l'écriture}

"Socrate, celui qui n'écrit pas", dit Nietzsche ${ }^{2}$. Cela est impossible. Socrate, il est vrai, ne pratique pas l'écriture textuelle. Mais tout le monde écrit: dans le silence, au moins, de l'oubli de soi. Car le soi est de l'écriture dans le langage même de l'inconscient. Mais toute image de soi est la dénégation de cette écriture: l'oubli au second degré, l'oubli de l'oubli

2 Cité par Jacques Derrida, De la grammatologie, Paris, Minuit, 1967, p. 15. 
de soi. La parole d'un je: le double de la mémoire. Je est le théâtre de la mort. "On périt toujours par le moi qu'on assume: porter un nom c'est revendiquer un mode exact d'effondrement", écrit Cioran ${ }^{3}$. L'identité est fatale.

Qui suis-je? L'écriture ne répond pas. Je me redéfinis chaque jour à cause d'elle, littéralement. Je suis vivant.

La vie est elle-même de l'autobiographie. Alors à quoi sert l'essentiel de ce à quoi l'on consacre sa vie, comme on dit? À se réaliser pleinement? Pourquoi cette doublure de l'écriture? Pourquoi laisser des traces à propos de ce qui n'en laisse pas? Pourquoi faire $s a$ marque? Quel impératif nous pousse ainsi à provoquer la mort? La réponse est simple: la vie ne suffit pas, il faut également en avoir le sentiment. Tout travail aurait d'abord ce sens-là. Le travail serait donc un moyen de se constituer un simulacre didentité: un je fendu et une image de soi fragile; aux bords flous. Un je aux remparts assiégés par la vie.

Le travail est une écriture au second degré. Écrire n'est don pas l'apanage des lettres. Mais il est difficile de le prétendre. Car le travail n'a pas échappé à la division en genres et en espèces. On donne à chacun un nom différent, réservant celui d'écriture au travail littéraire. Mais alors on masque la nature du travail, qui est écriture: faire siennes, momentanément, les marques d'une différence - s'approprier un signe -, l'échanger contre d'autres, en composer de nouvelles ou en défaire d'anciennes; et toujours pour se sentir vivre. Créer un monde pour soi, mais aussi turbulent et instable que soi, que lui. Se perdre à la limite des sources mêmes de la vie et se retenir juste à temps. Recommencer.

Je me plains comme un tout chaque fois que je me retrouve assez longtemps pour me reconnaître deux fois de suite. Alors je sais que j'ai cessé d'écrire. Et le sentiment de vivre fait place, peu à peu, à celui d'exister. Et c'est la descente aux enfers, l'expérience de la conscience malheureuse. La voie qu'emprunte le sujet pour se dépasser. Romantisme. Le travail reprend, mais il a changé de ton; et pour longtemps dans la tristesse.

Je préfere changer de voie; Hegel, c'est trop long. Ne reste plus qu'à rire de soi. Travailler contre l'image de soi: désarticuler le corps et composer des images sans pareilles. 
38

Le travail serait tératologique.

\section{L'imaginaire de soi}

La voie d'un imaginaire de soi est exigeante. Chaque fois que j'essaie de l'emprunter, l'ego résiste plus fort encore que les mots, les choses, les images ou les idées que lui oppose l'écriture au bas de laquelle l'ego, justement, veut apposer sa signature. La lecture et l'emprunt sont nécessaires à l'écriture tératologique; impossible de travailler seul contre soi. D'ailleurs, l'Autre est déjà le signataire de l'écriture biologique. L'enflure est l'absence de l'autre. Se prendre pour quelqu'un d'autre, ce n'est jamais qu'être complètement soi.

J'ai la tentation de l'Orient. Mais succomber serait un leurre: l'appropriation d'un discours que je n'aurais pas les moyens d'incarner. J'essaie quand même de me déployer un peu de ce côté-là. Ça fait du bien. Mais la lucidité, ainsi compromise, proteste en même temps avec des soubresauts de culpabilité. Je n'ai pas encore rejoint l'autre.

\section{$* * *$}

Méditant cela, j’ai dormi quelques heures avec mon fils. J'ai retrouvé le sentiment de vivre. Je n'ai pas signé cela. Je dormirais quelques heures avec toi.

Le passage même: au sommeil, au réveil, est une autre dimension de soi: transformations topologiques de l'ego; plis et traversion. Un imaginaire sans images. C'est sublime, au sens même de Kant: l'imagination atteint le vertige d'ellemême.

La classification de Borgès est un recensement sommaire des ébauches de soi. Elle atteint la perfection dans l'et cætera; tout l'imaginaire en une seule image impossible.

Un certain soi. Etc. 